\title{
The Associations Between Objectively Measured Gait Speed and Subjective Sleep Quality in First-Year University Students, According to Gender
}

\author{
Mario Kasović ${ }^{1,2}$ \\ Andro Štefan' \\ Lovro Štefan (iD) ${ }^{1-3}$ \\ 'Faculty of Kinesiology, Department of \\ General and Applied Kinesiology, \\ University of Zagreb, Zagreb, 10000 , \\ Croatia; ${ }^{2}$ Faculty of Sports Studies, \\ Department of Sport Motorics and \\ Methodology in Kinanthropology, \\ Masaryk University, Brno, 625 00, Czech \\ Republic; ${ }^{3}$ Faculty of Science, \\ Department of Recruitment and \\ Examination (RECETOX), Masaryk \\ University, Brno, 625 00, Czech Republic
}

Correspondence: Lovro Štefan Faculty of Kinesiology, Department of General and Applied Kinesiology, University of Zagreb, Zagreb, 10000 , Croatia

Tel +385-989l-77-060

Email lovro.stefan1510@gmail.com
Purpose: To examine the associations between gait speed and sleep quality in first-year university students, according to gender.

Methods: In this cross-sectional study, we recruited 193 first-year university students [mean age \pm standard deviation (SD): $19.6 \pm 1.1$ years; mean height: $178.0 \pm 10.5 \mathrm{~cm}$; mean weight: $74.0 \pm 11.0 \mathrm{~kg} ; 26.9 \%$ women). Sleep quality was assessed using the Pittsburgh Sleep Quality questionnaire, with a lower score indicating "better" sleep quality. Gait speed was measured using the Zebris pressure platform. The associations were examined with generalized linear models and multiple regression analysis.

Results: In the unadjusted model, faster participants had significantly "better" sleep quality $(\beta=-3.15,95 \%$ CI -3.82 to $-2.47, p<0.001)$. When the model was adjusted for sex, age, body-mass index, self-rated health, smoking status, and psychological distress, faster participants remained having "better" sleep quality $(\beta=-2.88,95 \% \mathrm{CI}-3.53$ to $-2.22, p<0.001)$. Conclusion: This study shows that sleep quality can be predicted by gait speed in the firstyear university students.

Keywords: youth, biomechanics, sleep, relationship

\section{Introduction}

Adequate sleep is one of the most important aspects of health. ${ }^{1-4}$ This statement does not surprise, since people spend almost one-third of their lifetime sleeping. ${ }^{5}$ It has been documented, that problems related to sleep quality mainly affect approximately $40 \%$ of young adults. ${ }^{6}$ Specifically, poor sleep quality may lead to healthrelated issues, including mental disorders ${ }^{7}$ or delinquent behaviors. ${ }^{8}$

Biomechanical characteristics of the gait reflect walking and moving patterns and can manifest health conditions. ${ }^{9,10}$ Since both sleep quality and gait are major contributors to health, one could expect that they influence each other. ${ }^{11}$ Previous evidence has highlighted, that the association between sleep quality and gait is bidirectional. $^{12-17}$ For example, a study by Goldman et $\mathrm{al}^{12}$ has shown, that the gait speed of those who sleep less than 6 hours per night is 3.5\% slower, compared to those who sleep 6-8 hours. A similar association has been confirmed by other researchers in older individuals. ${ }^{13}$ In clinical practice, poor sleep efficiency and greater sleep fragmentation were associated with the progression of step-width variability and a more rapid deterioration in gait for patients suffering from Parkinson's disease. ${ }^{14}$ Sleep quality has also been associated with daily energy expenditure, ${ }^{15}$ and daily energy expenditure has been highly associated with gait 
demands. ${ }^{16}$ Recently, a study by Liu et $\mathrm{al}^{11}$ has shown that gait can reveal sleep quality by using a machine learning model and Microsoft Kinect sensors in healthy students without disabilities.

According to the aforementioned, the associations between sleep quality and gait have been mostly conducted among older adults, ${ }^{12,13}$ patients, ${ }^{14-16}$ and general population, ${ }^{17,18}$ while studies in young adults are lacking. ${ }^{11}$ In general, sleeping problems start to occur in young adulthood, ${ }^{19}$ following several lifestyle changes of living home, going to university, and being independent. ${ }^{20,21}$

Therefore, the main purpose of the study was to examine the associations between gait speed and sleep quality in firstyear university students, according to gender. We hypothesized, that "slower" individuals would have "poorer" sleep quality, compared to those walking "faster". Also, women would experience more sleeping problems, while men would walk "faster". If the associations were significant, this would imply that sleep quality might be predicted by the level of gait speed and that future interventions aiming to improve sleep quality should also take gait speed into account.

\section{Materials and Methods}

\section{Study Participants}

In this cross-sectional study, we recruited a convenient sample of first-year university students from the Faculty of Kinesiology, University of Zagreb, Croatia. The total number of undergraduate first-year students at the time of measurement at the Faculty of Kinesiology was 230. By using a $95 \%$ confidence interval of $95 \%$ and a margin of error of $3 \%$, the appropriate sample size was calculated to be 190. The recruitment of the study participants is presented in Figure 1.Finally, 193 students had the questionnaires fulfilled and gait speed measured and were enrolled in future analyses. All students had to be healthy and without sleeping problems at the time of measurements, which was additionally explored by questionnaires. Before the study began, all participants had given a written informed consent to participate in the study. The procedures were anonymous and performed by the Declaration of Helsinki and approved by the Ethical Committee of the Faculty of Kinesiology, University of Zagreb, Croatia (Ethical code number: 2021).

\section{Sleep Quality}

Sleep quality was assessed using the Pittsburgh Sleep Quality Index, a questionnaire composed of 19 questions. $^{22}$ Each component is scored on a scale from 0 to 3 , where the lower value denotes no problems, while the higher value denotes worsening problems. When certain questions are combined, they create 7 major components in the following order: (1) subjective sleep quality, (2) sleep latency, (3) sleep duration, (4) sleep efficiency, (5) sleep disturbances, (6) use of sleeping medications and (7) daytime dysfunction. All seven components are then summed up to create a scale from 0 to 21 points. $^{22}$ The reliability of the Pittsburgh Sleep Quality Index in our

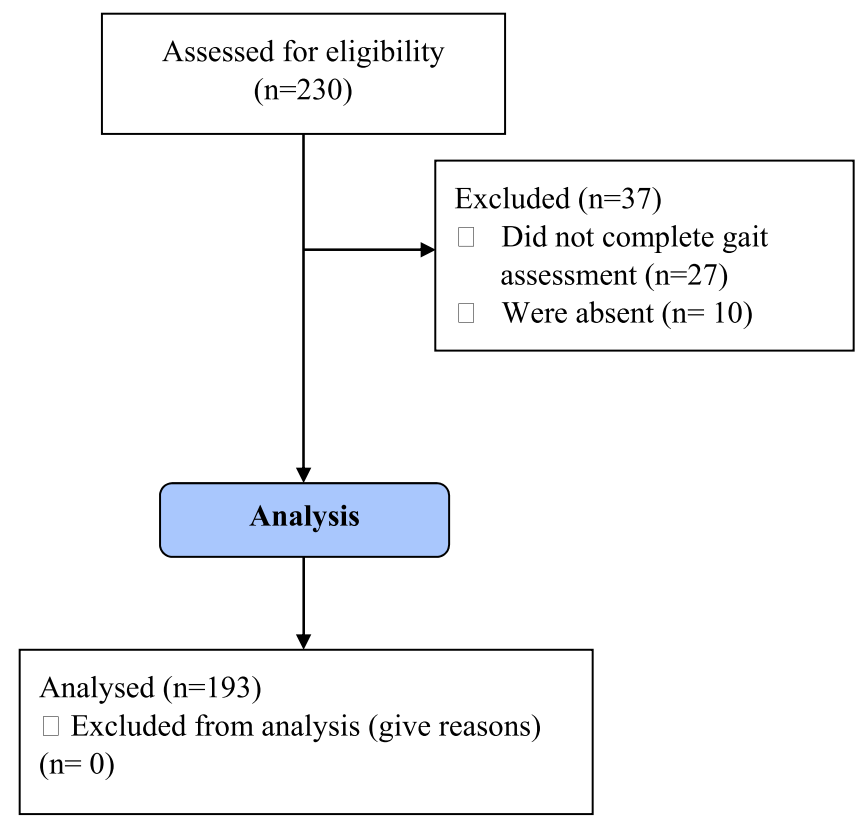

Figure I A flowchart diagram on the recruitment of the study participants. 
study was satisfactory (Cronbach's $\alpha=0.77$ ). Before entering the study, each participant was instructed not to consume alcohol at minimum of $48 \mathrm{~h}$ before the testing.

\section{Gait Speed}

To assess gait speed, we used a pressure platform (ZEBRIS company, FDM; GmbH, Munich, Germany; the number of sensors: 11,264; sampling rate: $100 \mathrm{~Hz}$; sensor area: $149 \mathrm{~cm} \times$ $54.2 \mathrm{~cm})$. The procedure of measuring gait speed has been described previously. ${ }^{23}$ In brief, the walkway was $10.5 \mathrm{~m}$ in length $(1.5 \mathrm{~m}$ platform and $4.5 \mathrm{~m}$ custom-designed dense material before and after the platform). The protocol consisted of a set of instructions to participants to walk at a preferred speed and to look straight forward across the platform being barefoot and without targeting the platform. When the participants finished walking across a $10.5 \mathrm{~m}$ walkway, they needed to turn around for $180^{\circ}$ and continue to walk over the platform until they reached the end of the walkway. The aforementioned protocol was repeated once again with a total of 6 trials across the platform. If we detected obvious gait deviations, we discarded the trials and repeated the protocol. $^{23}$ Of note, all measurements of gait speed were undertaken at the same time for 5 days (between 9:00 and 11:00 h).

\section{Covariates}

Self-rated health was assessed using a one-item question: "How would you rate your health?" with the following answers: (1) very poor, (2) poor, (3) fair, (4) good, and (5) excellent. ${ }^{24}$ Smoking status was categorized as (1) nonsmoker, (2) former smoker, and (3) present smoker. To assess the level of psychological distress, we used Kessler's six-item questionnaire with the following questions: (1) "How often during the past 30 days did you feel nervous?", (2) "How often during the past 30 days did you feel hopeless?", (3) "How often during the past 30 days did you feel restless or fidgety?", (4) "How often during the past 30 days did you feel so depressed that nothing could cheer you up?", (5) "How often during the past 30 days did you feel that everything was an effort?" and (6) "How often during the past 30 days did you feel worthless?". 25 Each question is scored from 0 (none of the time) to 4 (all of the time). Scores of each question are summed up between 0 and 24, with a lower score indicating a lower level of psychological distress. ${ }^{25}$ Height and weight were objectively measured by using an anthropometric kit and digital scale. Body-mass index was calculated using following formula: [weight $(\mathrm{kg}) / \mathrm{height}\left(\mathrm{m}^{2}\right)$ ].

\section{Data Analysis}

Basic descriptive statistics are presented as mean and SD for numerical and as percentages for categorical values. The Kolmogorov-Smirnov test showed that the data were normally distributed. Differences between men and women were examined using a Student's $t$-test for independent samples or a chisquare test. Pearson's coefficient was used to calculate the correlations between gait speed and sleep quality. The associations between gait speed and sleep quality were calculated with generalized linear models and multiple regression analysis. The dependent variable was sleep quality (score from 0-21) and the independent variable was gait speed. Sex, age, bodymass index, self-rated health, smoking status, and psychological distress served as covariates in the adjusted model. Beta coefficients $(\beta)$ with $95 \%$ CI were used to present the associations between sleep quality and gait speed. The significance was set at $p<0.05$. The Statistical Packages for Social Sciences version 24. (IBM Corporation, Chicago, Il) was used to calculate the data.

\section{Results}

Basic descriptive statistics are presented in Table 1 (mean age \pm SD: $19.6 \pm 1.1$ years; mean height: $178.0 \pm 10.5 \mathrm{~cm}$; mean weight: $74.0 \pm 11.0 \mathrm{~kg} ; 26.9 \%$ women). Men were taller, heavier, and had higher body-mass index values, compared to women. A higher prevalence of men was categorized as 'smokers', while women had significantly higher levels of psychological distress. Also, women experienced poorer sleep quality, compared to men. No significant differences in self-rated health and gait speed between the sexes were observed.

The associations between gait speed and sleep quality are presented in Table 2. In the unadjusted model, faster participants had significantly "better" sleep quality $(\beta=$ $-3.15,95 \% \mathrm{CI}-3.82$ to $-2.47, p<0.001)$. Among covariates put separately into the model, being a woman, being a smoker, and having higher psychological distress were all associated with "poorer" sleep quality. When the model was adjusted for sex, age, body-mass index, self-rated health, smoking status, and psychological distress, faster participants remained to have "better" sleep quality $(\beta=$ $-2.88,95 \% \mathrm{CI}-3.53$ to $-2.22, p<0.001)$. Unadjusted Pearson's coefficient revealed a moderate correlation between gait speed and sleep quality $(r=-0.55, p<0.001)$. When the correlation was adjusted for sex, age, body-mass index, self-rated health, smoking status, and psychological distress, gait speed remained moderately correlated to 
Table I Basic Descriptive Statistics of the Study Participants ( $N=193)$

\begin{tabular}{|c|c|c|c|c|}
\hline \multirow[t]{2}{*}{ Study Variables } & Total $(\mathrm{N}=193)$ & Men $(N=|4|)$ & Women $(\mathrm{N}=52)$ & \multirow[t]{2}{*}{$p$-value* } \\
\hline & Mean \pm SD & Mean士SD & Mean士SD & \\
\hline Age (years) & $19.6 \pm 1.1$ & $19.6 \pm 1.0$ & $19.5 \pm 1.5$ & 0.816 \\
\hline Height $(\mathrm{cm})$ & $178.0 \pm 10.5$ & $|8| .4 \pm 9.7$ & $168.6 \pm 6.1$ & $<0.001$ \\
\hline Weight (kg) & $74.0 \pm 11.0$ & $78.8 \pm 8.4$ & $61.0 \pm 5.4$ & $<0.001$ \\
\hline Body-mass index $\left(\mathrm{kg} / \mathrm{m}^{2}\right)$ & $23.1 \pm 2.1$ & $23.7 \pm 1.9$ & $21.5 \pm 1.7$ & $<0.001$ \\
\hline Self-rated health (scale I-5) & $4.2 \pm 0.7$ & $4.3 \pm 0.7$ & $4.2 \pm 0.8$ & 0.838 \\
\hline Smoking status (\% of "yes") & 11.4 & 12.8 & 7.7 & $<0.001$ \\
\hline Psychological distress (scale 0-24) & $5.8 \pm 3.6$ & $5.4 \pm 3.3$ & $7.0 \pm 4.0$ & 0.004 \\
\hline Sleep quality (scale 0-2I) & $9.0 \pm 4.0$ & $8.6 \pm 4.0$ & $10.1 \pm 4.1$ & 0.026 \\
\hline Gait speed $(\mathrm{km} / \mathrm{h})$ & $4.1 \pm 0.7$ & $4.1 \pm 0.7$ & $4.0 \pm 0.8$ & 0.536 \\
\hline
\end{tabular}

Note: *Denotes differences between sexes; $p<0.05$.

Table 2 The Associations Between Gait Speed and Sleep Quality ( $N=193)$

\begin{tabular}{|l|c|c|c|c|c|c|}
\hline \multirow{2}{*}{ Study Variables } & \multicolumn{3}{|c|}{ Model I* } & \multicolumn{2}{c|}{ Model 2** } \\
\cline { 2 - 6 } & $\boldsymbol{\beta}$ & $\mathbf{9 5 \%} \mathbf{~ C l}$ & p-value & $\beta$ & $95 \%$ Cl & $\boldsymbol{p}$-value \\
\hline Gait speed (km/h) & -3.15 & -3.82 to -2.47 & $<0.001$ & -2.88 & -3.53 to -2.22 & $<0.00$ I \\
Sex (I=men; 2=women) & 1.45 & 0.19 to 2.72 & 0.025 & 0.85 & -0.34 to 2.05 & 0.162 \\
Age (years) & 0.40 & -0.10 to 0.90 & 0.117 & 0.03 & -0.38 to 0.43 & 0.896 \\
Body-mass index (kg/m ${ }^{2}$ ) & 0.04 & -0.23 to 0.32 & 0.751 & 0.01 & -0.24 to 0.26 & 0.950 \\
Self-rated health (scale I-5) & -0.59 & -1.36 to 0.18 & 0.131 & 0.08 & -0.58 to 0.74 & 0.813 \\
Smoking status (\% of "yes") & 1.36 & -0.01 to 2.74 & 0.050 & 0.88 & -0.22 to 1.99 & 0.117 \\
Psychological distress (scale 0-24) & 0.37 & 0.22 to 0.52 & $<0.001$ & 0.25 & 0.11 to 0.40 & $<0.001$ \\
\hline
\end{tabular}

Notes: *Model I: examine the associations between gait speed and sleep quality, where sex, age, body-mass index, self-rated health, smoking status, and psychological distress were entered separately into the model; **Model 2: examine the associations between gait speed and sleep quality-adjusted for sex, age, body-mass index, self-rated health, smoking status, and psychological distress; $p<0.05$.

sleep quality $(r=-0.43, p<0.001)$. Of note, "slower" gait speed was correlated with "poorer" subjective sleep quality $(r=-0.36, p<0.001)$, "longer" sleep latency $(r=-0.40$, $p<0.001)$, "shorter" sleep duration $(r=-0.58, p<0.001)$, "poorer" sleep efficiency $(r=-0.34, p<0.001)$, "having more" sleep disturbances $(r=-0.40, p<0.001)$, "using" sleeping medications $(r=-0.21, p<0.001)$ and "greater" daytime dysfunction $(r=-0.60, p<0.001)$.

\section{Discussion}

The main purpose of the study was to examine the associations between gait speed and sleep quality in first-year university students. The main findings are: 1) women experience "poorer" sleep quality, compared to men; 2) gait speed is significantly associated with sleep quality and its components, that is "faster" participants have "better" sleep quality and 3) even after adjusting for potential covariates, similar associations between gait speed and sleep quality remain.
Our results are in line with previous findings conducted among young adults, ${ }^{11}$ general population ${ }^{17,18}$ and older adults. ${ }^{12,13}$ Specifically, a study by Liu et $\mathrm{al}^{11}$ has presented a new method for evaluating sleep quality in university students. By using Microsoft Kinect system to measure gait characteristics, the correlations between gait and sleep quality were high $(r=0.78)$, indicating that human health status may be predicted by using Microsoft Kinect. Another walking intervention study conducted among Japanese general population has shown, that walking improves global Pittsburgh Sleep Quality Index score, sleep latency (in minutes), sleep duration (in hours), perceived sleep quality and daily disturbance factor. ${ }^{19}$ In the same study, similar improvements were observed in both exercising and non-exercising group, pointing out that non-intentional light physical activity, such as walking, may have beneficial effects on sleep. ${ }^{19}$ Similar associations have been obtained for older adults, ${ }^{12,13}$ where short nighttime sleep and disrupted sleep are associated with poorer neuromuscular performance and more functional limitations. Finally, studies conducted in clinical practice among cataract patients 
have shown, that both sleep quality and gait significantly improved after the surgery, highlighting the importance of targeting both parameters, when improving overall health status. $^{15,16}$

Both sleep quality and gait have been consistently associated with health. ${ }^{2-4,10}$ However, the mechanism underlying the associations between sleep quality and gait is still unknown. It has been reported that the control of the gait is under automatic and higher executive control systems, ${ }^{26}$ where sleep abnormalities lead to more gait deviations, subsequently leading to slower processing and alternations in gait patterns. ${ }^{27}$ Another explanation points out that gait neuroanatomical regions and sleep-regulation centers are connected by different nuclei areas controlling gait, including the pontine tegmentum, the pedunculopontine nucleus, and the medial medulla. ${ }^{28}$ Nevertheless, "poor" sleep quality and "slower" gait speed $(<1 \mathrm{~m} / \mathrm{s})$ increase the risk of morbidity and premature mortality, ${ }^{3,10}$ and intervention studies aiming to improve one health indicator would indirectly affect the other one and vice versa.

This study is not without limitations. First, we used a crosssectional design, so we cannot exclude the possibility of reverse causality, that is, "slow" gait speed led to "poor" sleep quality. Second, we used subjective measures to assess sleep quality and general health. Self-report measures tend to lead to considerable measurement error, recall bias and social desirability effect, ${ }^{29}$ and therefore there is a possibility of common method bias which may have resulted in bias away from the null. Moreover, students completed the questionnaires at the individual level, referring to the students' individual perception of sleep quality. Third, no sleeping diaries were assessed prior the testing. However, one criterium for entering the study was to have healthy sleeping patterns, so we believe that none of the participants suffered from sleeping problems. Fourth, we only recruited the first-year university students with more than $70 \%$ men, which might have influenced the results. Finally, an early morning testing could have induced a a night of poor sleep in evening types. However, they were instructed not to consume any alcohol $48 \mathrm{~h}$ prior the testing. Also, no oral or written exams had been performed at the time of testing, so we can rule out studying late at night. Moreover, daily faculty obligations usually start at 8:00 am for all students, and we can speculate that evening type students had already adjusted their sleeping patterns, according to faculty timetable. Therefore, future research should use a longitudinal, population-based design with objective methods (by using actigraphy or polysomnography to assess sleep duration and sleep quality and more detailed medical check-up to assess general health) and greater age range, to explore the associations between gait speed and sleep quality.

\section{Conclusions}

This study shows a significant association between sleep quality and gait in healthy first-year university students, according to gender, Also, no significant differences in gait speed between gender is observed, yet women experience more sleeping problems, compared to men. Therefore, healthrelated professionals should use both gait speed and sleep quality in men and women, when establishing health status of individuals, that is sleep quality is predicted by the level of gait speed and strategies based on sleep quality improvements should also take gait speed, as a potential factor.

\section{Data Sharing Statement}

All the data are freely available upon reasonable request from the corresponding author.

\section{Ethics Approval}

Ethical Committee of the Faculty of Kinesiology.

\section{Consent to Participate}

Written obtained.

\section{Acknowledgments}

We would like to thank all the study participants for their enthusiastic participation in the study.

\section{Author Contributions}

All authors made a significant contribution to the work reported, whether that is in the conception, study design, execution, acquisition of data, analysis and interpretation, or in all these areas; took part in drafting, revising or critically reviewing the article; gave final approval of the version to be published; have agreed on the journal to which the article has been submitted; and agree to be accountable for all aspects of the work.

\section{Funding}

This research did not receive any specific grant from funding agencies in the public, commercial, or not-forprofit sectors.

\section{Disclosure}

The authors report no conflicts of interest in this work. 


\section{References}

1. Laposky AD, Van Cauter E, Diez-Roux AV. Reducing health disparities: the role of sleep deficiency and sleep disorders. Sleep Med. 2016;18:3-6. doi:10.1016/j.sleep.2015.01.007

2. Kanagasabai T, Chaput J-P. Sleep duration and the associated cardiometabolic risk scores in adults. Sleep Health. 2017;3(3):195-203. doi:10.1016/j.sleh.2017.03.006

3. Marshall NS, Wong KK, Cullen SR, Knuiman MW, Grunstein RR. Sleep apnea and 20-year follow-up for all-cause mortality, stroke, and cancer incidence and mortality in the Busselton Health Study cohort. $J$ Clin Sleep Med. 2014;10(04):355. doi:10.5664/jcsm.3600

4. Sigurdson K, Ayas NT. The public health and safety consequences of sleep disorders. Can J Physiol Pharmacol. 2007;85(1):179-183. doi:10.1139/y06-095

5. Hirshkowitz M, Whiton K, Albert SM, et al. National sleep foundation's sleep time duration recommendations: methodology and results summary. Sleep Health. 2015;1(1):40-43. doi:10.1016/j.sleh.2014.12.010

6. Centers for Disease and Control Prevention. Morbidity and mortality weekly report. $M M W R$. 2011;60:233-256.

7. Glozier N, Martiniuk A, Patton G, et al. Short sleep duration in prevalent and persistent psychological distress in young adults: the DRIVE study. Sleep. 2010;33(9):1139-1145. doi:10.1093/sleep/ 33.9.1139

8. Clinkinbeard SS, Simi P, Evans MK, Anderson AL. Sleep and delinquency: does the amount of sleep matter? J Youth Adolesc. 2011;40 (7):916-930. doi:10.1007/s10964-010-9594-6

9. Sun B, Zhang Z, Liu X, Hu B, Zhu T. Self-esteem recognition based on gait pattern using Kinect. Gait Posture. 2017;58:428-432. doi:10.1016/j.gaitpost.2017.09.001

10. Kramer RS, Gottwald VM, Dixon TA, Ward R. Different cues of personality and health from the face and gait of women. Evol Psychol. 2012;10(2):147470491201000208. doi:10.1177/14747049 1201000208

11. Liu X, Sun B, Zhang Z, Wang Y, Tang H, Zhu T. Gait can reveal sleep quality with machine learning models. PLoS One. 2019;14(9): e0223012. doi:10.1371/journal.pone. 0223012

12. Goldman SE, Stone KL, Ancoli-Israel S, et al. Poor sleep is associated with poorer physical performance and greater functional limitations in older women. Sleep. 2007;30(10):1317. doi:10.1093/sleep/ 30.10 .1317

13. Agmon M, Shochat T, Kizony R. Sleep quality is associated with walking under dual-task, but not single-task performance. Gait Posture. 2016;49:127-131. doi:10.1016/j.gaitpost.2016.06.016

14. O’Dowd S, Galna B, Morris R, et al. Poor sleep quality and progression of gait impairment in an incident Parkinson's disease cohort. J Parkinsons Dis. 2017;7(3):465-470. doi:10.3233/JPD-161062

15. Ayaki M, Negishi K, Tsubota K. Rejuvenation effects of cataract surgery with ultraviolet blocking intra-ocular lens on circadian rhythm and gait speed. Rejuvenation Res. 2014;17(4):359-365. doi:10.1089/rej.2014.1550
16. Ayaki M, Muramatsu M, Negishi K, Tsubota K. Improvements in sleep quality and gait speed after cataract surgery. Rejuvenation Res. 2013;16(1):35-42. doi:10.1089/rej.2012.1369

17. Waters RL, Mulroy S. The energy expenditure of normal and pathologic gait. Gait Posture. 1999;9(3):207-231. doi:10.1016/S09666362(99)00009-0

18. Hori H, Ikenouchi-Sugita A, Yoshimura R, Nakamura J. Does subjective sleep quality improve by a walking intervention? A real-world study in a Japanese workplace. BMJ Open. 2016;6(10):e011055. doi:10.1136/bmjopen-2016-011055

19. Matthews T, Danese A, Gregory AM, Caspi A, Moffitt TE, Arseneault L. Sleeping with one eye open: loneliness and sleep quality in young adults. Psychol Med. 2017;47(12):2177-2186. doi:10.1017/S0033291717000629

20. Maslowsky J, Ozer EJ. Developmental trends in sleep duration in adolescence and young adulthood: evidence from a national United States sample. J Adolesc Health. 2014;54(6):691-697. doi:10.1016/j. jadohealth.2013.10.201

21. Quick V, Byrd-Bredbenner C, Shoff S, et al. Relationships of sleep duration with weight-related behaviors of U.S. college students. Behav Sleep Med. 2016;14(5):565-580. doi:10.1080/1540200 2.2015.1065411

22. Buysse DJ, Reynolds CF 3rd, Monk TH, Berman SR, Kupfer DJ. The Pittsburgh sleep quality index: a new instrument for psychiatric practice and research. Psychiatry Res. 1989;28(2):193-213. doi:10.1016/0165-1781(89)90047-4

23. Kasović M, Štefan L, Borovec K, Zvonař M, Cacek J. Effects of carrying police equipment on spatiotemporal and kinetic gait parameters in first-year police officers. Int J Environ Res Public Health. 2020;17(16):5750. doi:10.3390/ijerph17165750

24. Larsson D, Hemmingsson T, Allebeck P, et al. Self-rated health and mortality among young men: what is the relation and how may it be explained? Scand J Public Health. 2002;30(4):259-266. doi:10.1080/ 14034940210133997

25. Kessler RC, Barker PR, Colpe LJ, et al. Screening for serious mental illness in the general population. Arch Gen Psychiatry. 2003;60 (2):184-189. doi:10.1001/archpsyc.60.2.184

26. Clark DJ. Automaticity of walking: functional significance, mechanisms, measurement and rehabilitation strategies. Front Hum Neurosci. 2015;9:246. doi:10.3389/fnhum.2015.00246

27. Schneider W, Chein JM. Controlled \& automatic processing: behavior, theory, and biological mechanisms. Cogn Sci. 2003;27 (3):525-559. doi:10.1207/s15516709 $\operatorname{cog} 2703 \_8$

28. Lewis SJ. Neurological update: emerging issues in gait disorders. $J$ Neurol. 2015;262:1-6.

29. Shepard RJ. Limits to the measurement of habitual physical activity by questionnaires. Br J Sports Med. 2003;37:197-206. doi:10.1136/ bjsm.37.3.197
Nature and Science of Sleep

\section{Publish your work in this journal}

Nature and Science of Sleep is an international, peer-reviewed, open access journal covering all aspects of sleep science and sleep medicine, including the neurophysiology and functions of sleep, the genetics of sleep, sleep and society, biological rhythms, dreaming, sleep disorders and therapy, and strategies to optimize healthy sleep.
The manuscript management system is completely online and includes a very quick and fair peer-review system, which is all easy to use. Visit http://www.dovepress.com/testimonials.php to read real quotes from published authors. 